\title{
Hearing Preservation after Low-dose Gamma Knife Radiosurgery of Vestibular Schwannomas
}

\author{
Ayako HORIBA, ${ }^{1}$ Motohiro HAYASHI, ${ }^{1,2}$ Mikhail CHERnOv, ${ }^{1,2}$ \\ Takakazu KAWAMATA, ${ }^{1}$ and Yoshikazu OKADA ${ }^{1}$
}

\author{
Department of Neurosurgery ${ }^{1}$ and Faculty of Advanced Techno-Surgery, ${ }^{2}$ \\ Tokyo Women's Medical University, Shinjuku, Tokyo
}

\begin{abstract}
The objective of the retrospective study was to evaluate the factors associated with hearing preservation after low-dose Gamma Knife radiosurgery (GKS) of vestibular schwannomas performed according to the modern standards. From January 2005 to September 2010, 141 consecutive patients underwent such treatment in Tokyo Women's Medical University. Mean marginal dose was 11.9 Gy (range, 11-12 Gy). The doses for the brain stem, cranial nerves (V, VII, and VIII), and cochlea were kept below 14 Gy, 12 Gy, and $4 \mathrm{~Gy}$, respectively. Out of the total cohort, 102 cases with at least 24 months follow-up were analyzed. Within the median follow-up of 56 months (range, 24-99 months) the crude tumor growth control was $92 \%$ (94 cases), whereas its actuarial rate at 5 years was $93 \%$. Out of 49 patients with serviceable hearing on the side of the tumor before GKS, 28 (57\%) demonstrated its preservation at the time of the last followup. No one evaluated factor, namely Gardner-Robertson hearing class before irradiation, Koos tumor stage, extension of the intrameatal part of the neoplasm up to fundus, nerve of tumor origin, presence of cystic changes in the neoplasm, and cochlea dose demonstrated statistically significant association with preservation of the serviceable hearing after radiosurgery. In conclusion, GKS of vestibular schwannomas performed according to the modern standards of treatment permits to preserve serviceable hearing on the side of the tumor in more than half of the patients. The actual causes of hearing deterioration after radiosurgery remain unclear.
\end{abstract}

Key words: Gamma Knife radiosurgery, hearing preservation, outcome, vestibular schwannoma

\section{Introduction}

At present, Gamma Knife radiosurgery (GKS) represents widely approved management option for vestibular schwannomas of small and medium size. Application of the modern treatment standards, particularly use of low-dose irradiation (marginal dose 11-13 Gy at $50 \%$ isodose line), results in $89-99 \%$ crude tumor control rates, ${ }^{1-15)}$ which reported actuarial rates at 5 years and 10 years after GKS constitutes 93-95\% and $86-95 \%$, respectively. ${ }^{2,11-13)}$ Approximately two-thirds of the neoplasms undergo more or less prominent shrinkage. ${ }^{8,11-15)}$ Conformal and selective irradiation provided by the robotized devices, such as automatic positioning system (APS) and Perfexion $^{\mathrm{TM}}$ (Elekta Instruments AB, Stockholm, Sweden), minimizes the risk of treatment-related complications, including trigeminal and facial neuropathy.

Received August 10, 2015; Accepted January 18, 2016
Nevertheless, possible deterioration of hearing after GKS of vestibular schwannomas represents the main concern for its application, particularly in younger patients. The actuarial preservation rates of serviceable hearing after radiosurgery constitute $47-77 \%$, $28-64 \%$, and $23-45 \%$ at 3 years, 5 years, and 10 years, respectively. ${ }^{2,6,12,16-22)}$ Yang et al. ${ }^{23)}$ performed a comprehensive analysis of 45 published studies incorporating 4,234 patients, who underwent GKS for vestibular schwannomas with a median follow-up time among different series of 35 months; serviceable hearing was preserved in $60.5 \%$ and $50.4 \%$ of cases if marginal dose was < 13 Gy or $>13 \mathrm{~Gy}$ $(\mathrm{P}=0.0005)$. Systemic meta-analysis performed by Arthurs et al. ${ }^{8)}$ revealed $60 \%$ hearing preservation rate among 1,850 included patients treated with a mean marginal dose of 12.6 Gy and followed in average 71 months after irradiation. In our recent investigation $77 \%$ of patients with serviceable hearing before irradiation maintained it at 3 years after GKS, whereas trend for hearing deterioration 
was revealed as early as 6 months after treatment. ${ }^{15)}$ The objective of the present retrospective study was evaluation of factors associated with preservation of hearing after GKS performed according to the modern treatment standards.

\section{Materials and Methods}

From January 2005 to September 2010, 141 consecutive patients with vestibular schwannomas were treated according to the previously described concept of robotic Gamma Knife microradiosurgery. ${ }^{24)}$ Shortly, on the day of treatment, Leksell $G$ stereotactic frame (Elekta Instruments $A B$ ) was attached to the patient's head under local anesthesia. Both computed tomography (CT) and magnetic resonance imaging (MRI) were attained routinely under stereotactic conditions. Axial CT (slice thickness $1.0 \mathrm{~mm}$ ) always included "bone window" images. The original MRI protocol included axial plain and gadoliniumenhanced constructive interference in steady state (CISS) images (slice thickness $0.5 \mathrm{~mm}$ ) and modified time-of-flight (TOF) images (slice thickness $1.0 \mathrm{~mm}$ ). Fusion of CT and MRI images was done within Leksell GammaPlan (Elekta Instruments AB). All patients were treated with the use of Leksell Gamma Knife model 4C with APS (Elekta Instruments AB). During treatment planning, the main emphasis was put on clear identification of the tumor borders and adjacent cranial nerves. Multiple small-sized isocenters were applied with a goal to attain both conformal and selective coverage of the lesion with $50 \%$ isodose. Conformity and selectivity indexes ${ }^{25}$ were usually kept $>0.95$ and $>0.90$, respectively. All isocenters were preferentially located within the border of the neoplasm with minimal involvement of the adjacent anatomical structures (Fig. 1). Mean marginal dose was 11.9 Gy (range, 11-12 Gy). The doses for brain stem, cranial nerves (V, VII, and VIII), and cochlea were generally kept below 14 Gy, $12 \mathrm{~Gy}$, and $4 \mathrm{~Gy}$, respectively. ${ }^{24)}$

Out of the total cohort, 102 cases with at least 2 years follow-up after treatment were selected for the present retrospective analysis, which was approved by the Ethics Committee of the Tokyo Women's Medical University (\#3505; July 30, 2015). In total, 49 patients $(48 \%)$ had serviceable hearing before treatment, corresponding to Gardner-Robertson classes ${ }^{26)}$ I (22 cases) or II (27 cases). According to Koos topographical classification, ${ }^{27)} 14$ (14\%) schwannomas corresponded to stage I ("intracanalicular"), 40 (39\%) were stage II ("protruding into cerebellopontine angle"), 26 (25\%) were stage III ("touching brain stem"), and 22 (22\%) were stage IV ("compressing brain stem"). Detailed evaluation of the local neuroanatomy with the use of
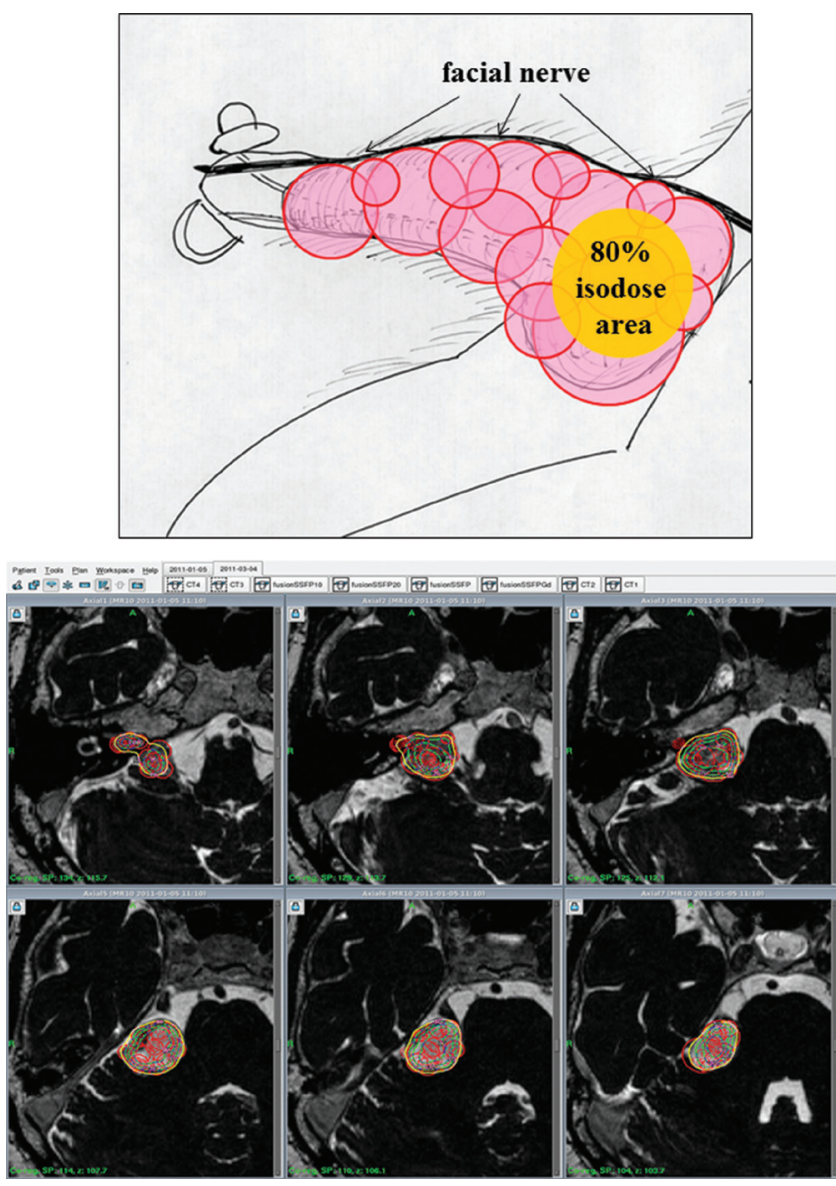

Fig. 1 Schematic (upper) and actual (lower) treatment planning for Koos grade III right-sided vestibular schwannoma based on the concept of robotic Gamma Knife microradiosurgery. Note use of multiple small-sized isocenters providing conformal and selective coverage of the lesion with $50 \%$ prescription isodose line, limited irradiation of the anterior border of the tumor for preventing facial nerve injury, and avoidance of the extending the $80 \%$ isodose area on the intracanalicular part of the neoplasm. From Hayashi et al. ${ }^{24)}$

Leksell GammaPlan revealed that 74 tumors (72\%) originated from the superior vestibular nerve, 19 $(19 \%)$ from the inferior vestibular nerve, and $3(3 \%)$ from the cochlear nerve, whereas in 6 cases $(6 \%)$ the exact nerve of tumor origin was not identified. In 33 cases (32\%), the internal acoustic canal was fully packed by the intrameatal part of the neoplasm extending up to fundus. Intra- or peritumoral cystic changes were noted in 14 cases.

\section{Follow-up}

The patients were discharged from the hospital next day after radiosurgery. Thereafter they were followed by attending neurosurgeon in the outpatient clinic of the Tokyo Women's Medical University. Clinical 
examinations and contrast-enhanced MRI were performed each 6 months during 2 years after treatment and yearly thereafter. Tumor volume during follow-up was calculated on gadolinium-enhanced $\mathrm{T}_{1}$-weighted images according to the following formula: largest length $(\mathrm{cm}) \times$ largest height $(\mathrm{cm}) \times$ largest width $(\mathrm{cm}) \times 0.5$. Enlargement or shrinkage of the neoplasm was defined as at least 10\% change of its volume, compared to the baseline data determined at the time of radiosurgery. Deterioration of hearing was defined as downgrading of at least one Gardner-Robertson class. The length of follow-up varied from 24 months to 99 months (mean, 55 months; median 56 months).

\section{Results}

Overall, tumor growth control was attained in 94 cases (92\%). Actuarial tumor growth control rates at 3 years and 5 years after GKS were $98 \%$ and $93 \%$, respectively. Three patients $(3 \%)$ required additional treatment after initial GKS (microsurgical resection, two cases; second GKS, one case). In 86 cases $(84 \%)$, shrinkage of the lesion was noted and in 60 cases $(59 \%)$ mass volume reduction was $>50 \%$. Temporary deterioration of the motor function of the facial nerve after irradiation was marked in one patient $(1 \%)$. There was no one case of trigeminal neuropathy after radiosurgery.

Overall, deterioration of hearing was noted in 31 patients $(30 \%)$. Out of 49 patients with serviceable hearing on the side of the tumor before GKS, 28 $(57 \%)$ demonstrated its preservation at the time of the last follow-up (Fig. 2). Out of 21 patients who lost serviceable hearing after GKS, it had happened within 1,2 , and 3 posttreatment years in $6(29 \%)$, $14(67 \%)$, and $18(86 \%)$ cases, respectively. In cases with and without extension of the intrameatal part of the neoplasm up to fundus, preservation of the serviceable hearing was attained in $40 \%$ and $60 \%$ of cases, respectively, however the difference did not reach statistical significance (odds ratio, 2.6; 95\% confidence interval, 0.5-12.4). Similarly, no one other investigated factor, namely Gardner-Robertson hearing class before irradiation, Koos tumor stage, nerve of tumor origin, presence of cystic changes in the neoplasm, and cochlea dose demonstrated statistically significant association with preservation of the serviceable hearing after GKS.

\section{Discussion}

Our concept of robotic Gamma Knife microradiosurgery was developed for highly conformal and selective management of the intracranial lesions with avoidance of excessive irradiation of the adjacent

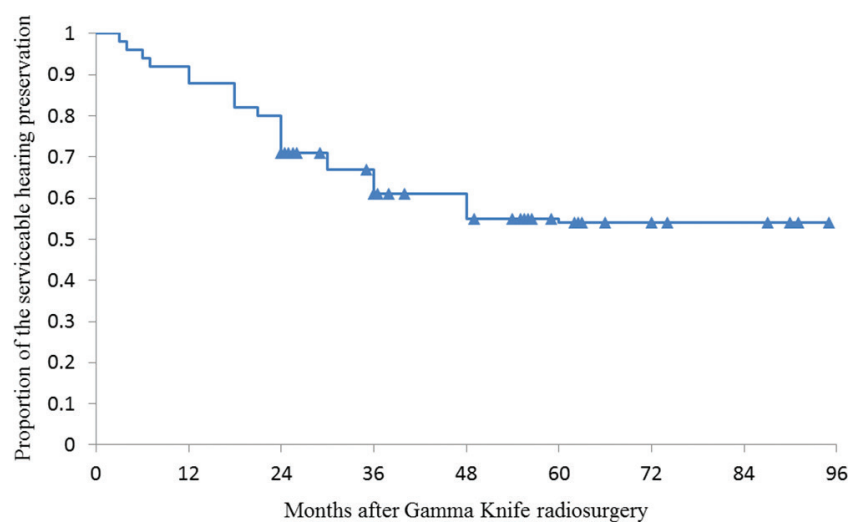

Fig. 2 Preservation of the serviceable hearing after low-dose Gamma Knife radiosurgery for vestibular schwannomas in the present series. Triangles mark censored observations.

anatomical structures based on advantages of the robotized devices for GKS (APS and Perfexion ${ }^{\mathrm{TM}}$ ). ${ }^{15,24)}$ Treatment planning is mainly based on high-resolution CISS images, which provide superior stereotactic definition of the target, particularly identification of the cranial nerves. ${ }^{28)}$ Local neuroanatomy is addressed in all details. Fused CT/MRI images are used routinely for avoidance of mislocalization errors caused by distortion artifacts. ${ }^{29)}$ For dose planning multiple small-sized isocenters carefully positioned within the border of the neoplasm are used. With a specific goal to attain tumor shrinkage wide $80 \%$ prescription isodose area is created in the center of the mass, while keeping sufficiently low marginal dose for prevention of complications. In our experience such treatment strategy in cases of various benign skull base neoplasms provided tumor control and shrinkage rates of $98 \%$ and $75 \%$, respectively, ${ }^{24)}$ whereas in the present series these parameters were $92 \%$ and $84 \%$. While it is still questionable whether volumetric response of vestibular schwannomas to GKS is related to dosimetric parameters, ${ }^{10)}$ highly selective and conformal irradiation definitely results in reduction of treatmentassociated morbidity, which in our series has been minimal. Applying similar radiosurgical principles others also marked improved clinical outcomes after management of vestibular schwannomas., ${ }^{3,30)}$ Nevertheless, according to results of the present study it seems that applied advanced treatment concept has low impact on hearing preservation rates after GKS. It corroborates to results of Massager et al. ${ }^{10)}$ who did not find associations between main indexes of dose distribution and audiological outcome.

The mechanisms of hearing deterioration after radiosurgery for vestibular schwannomas generally remain obscured. Among multiple associated factors, 
dose delivered to the cochlea is usually considered as the most relevant. ${ }^{4,6,17,31-34)}$ In the present series, the dose delivered to the cochlea was generally kept below 4 Gy and preservation of the serviceable hearing after GKS was attained in $57 \%$ of patients within the median follow-up of 56 months. It well corresponds to results reported in the recent literature (Table 1). ${ }^{1-7,9,10,14,15,18,19,22,34)}$ Of note, there is some evidence that impact of the cochlear dose on the hearing preservation after GKS of vestibular schwannomas may be not as strong as usually considered. First, in several studies applied multivariate statistical analysis did not reveal independent association between loss of serviceable hearing and cochlear dose. $^{4,14,18,21,22)}$ Second, in cases of other cerebellopontine tumors, such as meningiomas ${ }^{35)}$ and facial nerve schwannomas, ${ }^{36)}$ hearing loss after GKS is relatively rare despite frequent delivery of the cochlear doses in excess of 7-10 Gy, which is roughly 2-2.5 times greater than "safe dose" of 4 Gy recommended for optimal audiological outcome after radiosurgery of vestibular schwannomas. ${ }^{34)}$ It suggests that hearing deterioration after irradiation might be, in fact, multifactorial, but its actual causes remain unclear.

Other previously reported favorable prognostic factors for hearing preservation after radiosurgery of vestibular schwannomas include younger age of the patient, ${ }^{3,4,6,14)}$ initial symptom other than hearing deterioration, ${ }^{6)}$ hearing function corresponding to Gardner-Robertson class I or AAO-HNS class $\mathrm{A},{ }^{3,4,7,12,14,17,19,21,22)}$ absence of subjective hearing loss in patients with Gardner-Robertson class I hearing, ${ }^{37)}$ normal results of pure tone audiometry ${ }^{4,18,21,22,37)}$, speech discrimination ${ }^{4,22)}$ and auditory brainstem response $^{7,18)}$ before treatment, smaller size of the tumor $^{3,4,6,21,23)}$ and its less prominent extension into the internal auditory canal, ${ }^{6,31,32)}$ lower doses to the cochlear nuclei in the brainstem, ${ }^{33)}$ cochlear nerve ${ }^{33)}$ and intracanalicular part of schwannoma, ${ }^{31,32)}$ and absence of the transient tumor enlargement after irradiation. ${ }^{19)}$ Administration of steroids at the time of hearing impairment after GKS was suggested as possible preventive measure for hearing loss, while it was not statistically proved. ${ }^{19)}$ Anyway, in our patients no one evaluated factor demonstrated statistically significant association with the audiological outcome.

In the present study, special emphasis was put on evaluation of the interrelationships between the tumor extension within the internal acoustic canal and hearing preservation after GKS. Previously, Massager et al. ${ }^{31,32)}$ found significant association of

Table 1 Hearing preservation after low-dose Gamma Knife radiosurgery for unilateral vestibular schwannomas

\begin{tabular}{|c|c|c|c|}
\hline Author, year of publication & Marginal dose (Gy) & Length of follow-up (mos) & $\begin{array}{l}\text { Maintenance of the } \\
\text { serviceable hearing }\end{array}$ \\
\hline Pollock et al., 2006 ${ }^{1)}$ & mean, 12.2 & 12-62 (mean, 42) & $63 \%$ \\
\hline Chopra et al., 2007²) & 12-13 (median, 13) & median, 68; maximum, 143 & $57 \%$ \\
\hline Régis et al., 2007³) & 12 & at least, 24 & $60 \%$ \\
\hline Kano et al., 20094) & 12-13 (median, 12.5) & 6-40 (median, 20) & $71 \%$ \\
\hline Myrseth et al., 20095) & 12 & 24 & $68 \%$ \\
\hline Tamura et al., 20096) & 9-13 (median, 12) & 36-132 (median, 48) & $78 \%$ \\
\hline Kim et al., 2010 & 11-15 (median, 12) & 9-81 (mean, 36) & $68 \%$ \\
\hline Delbrouck et al., 20119) & 12 & at least, 12 & $66 \%$ \\
\hline Kim et al., 2011 19 ) & 12-13 (median, 12) & $6-48($ mean, 25) & $61 \%$ \\
\hline Massager et al., 2011 ${ }^{10)}$ & 12 & 24-96 (mean, 43) & $79 \%$ \\
\hline Han et al., 2012 ${ }^{18)}$ & 11-20 (median, 12) & 12-158 (mean, 55) & $57 \%$ \\
\hline Yomo et al., 2012 & 9-14 (mean, 12.1) & 7-123 (mean, 52) & $58 \%$ \\
\hline Boari et al., 2014 ${ }^{14)}$ & 11-15 (median, 13) & 36-153 (mean, 59.9) & $49 \%$ \\
\hline Jacob et al., 2014 22$)$ & 12-13 (median, 12) & 7-46 (median, 25) & $64 \%$ \\
\hline Lipski et al., 2015 & 11-12 (mean, 11.5) & 24-84 (median, 48) & $77 \%$ \\
\hline Present series & 11-12 (mean, 11.9) & 24-99 (median, 56) & $57 \%$ \\
\hline
\end{tabular}

Mos: months.

Neurol Med Chir (Tokyo) 56, April, 2016 
the audiological outcome with the volume of the intracanalicular tumor and amount of radiation energy (integrated dose) delivered to it. In their study, patients with intracanalicular tumor volume $>100 \mathrm{~mm}^{3}$ were 3.5 times more likely to experience hearing deterioration, whereas those who received $<1.5 \mathrm{~mJ}$ to the intracanalicular tumor were 16.7 times more likely to have preserved hearing. ${ }^{31)}$ However, in their cohort cochlear dose was also significantly associated with the audiological outcome and there was strong correlation between mean cochlear dose and amount of radiation energy delivered to the intracanalicular tumor. ${ }^{32}$ Because of the limited number of cases the interrelations between three investigated variables (intracanalicular tumor volume, amount of radiation energy delivered to it, cochlear dose) could not be accurately evaluated with a multivariate statistical analysis. ${ }^{31,32)}$ In the whole cohort of our patients hearing loss before treatment was associated with full packing of the internal acoustic canal by the intrameatal part of schwannoma (data not shown). Such tumor extension up to fundus was also associated with slightly worse preservation of the serviceable hearing after GKS, but the difference did not reach statistical significance.

Despite gradual deterioration of audiological function after irradiation, it should be noted that, at present, radiosurgery seemingly provides the best possible option for preservation of the serviceable hearing in cases of vestibular schwannomas. Régis et al. ${ }^{16)}$ compared 47 patients with intracanalicular tumors under wait-and-see strategy to 34 individuals who underwent proactive GKS. The chances of maintaining functional hearing and avoiding further intervention were much higher in the treated group $\left(60 \%\right.$ vs. $14 \%$ at 5 years of follow-up). ${ }^{16)}$ While in the surgical series hearing preservation has been reportedly attained in $50-63 \%$ of eligible patients with vestibular schwannomas, ${ }^{38,39)}$ both retrospective $^{40)}$ and prospective ${ }^{1,5)}$ comparisons of GKS and microsurgical resection demonstrated better audiological outcomes after radiosurgery. In the recent systematic review hearing preservation rates after observation, radiosurgery, and microsurgery for vestibular schwannomas were $58.9 \%, 60.2 \%$, and $4.3 \%$, respectively, whereas corresponding rates of tumor controls were $71.1 \%, 97.0 \%$, and $94.3 \% .{ }^{41)}$ Therefore, importance of the hearing deterioration after GKS of vestibular schwannomas should not be overemphasized.

\section{Conclusion}

The present study demonstrated that low-dose GKS performed according to the modern standards of treatment allows to preserve serviceable hearing on the side of the tumor in more than half of patients with vestibular schwannoma within the medianrange follow-up. It was not possible to identify any statistically significant prognostic factor for audiological outcome and the actual causes of hearing deterioration after irradiation remain unclear.

\section{Acknowledgments}

The results of this study were presented during the $17^{\text {th }}$ International Meeting of the Leksell Gamma Knife Society, May 11-15, 2014, New York, USA and at the $12^{\text {th }}$ International Stereotactic Radiosurgery Society Congress, June 7-11, 2015, Yokohama, Japan.

\section{Conflicts of Interest Disclosure}

This study contains no conflict of interests.

\section{References}

1) Pollock BE, Driscoll CLW, Foote RL, Link MJ, Gorman DA, Bauch CD, Mandrekar JN, Krecke KN, Johnson $\mathrm{CH}$ : Patient outcomes after vestibular schwannoma management: a prospective comparison of microsurgical resection and stereotactic radiosurgery. Neurosurgery 59: 77-85; discussion 77-85, 2006

2) Chopra R, Kondziolka D, Niranjan A, Lunsford LD, Flickinger JC: Long-term follow-up of acoustic schwannoma radiosurgery with marginal tumor doses of 12 to 13 Gy. Int J Radiat Oncol Biol Phys 68: 845-851, 2007

3) Régis J, Roche PH, Delsanti C, Thomassin JM, Ouaknine M, Gabert K, Pellet W: Modern management of vestibular schwannomas. Prog Neurol Surg 20: 129-141, 2007

4) Kano H, Kondziolka D, Khan A, Flickinger JC, Lunsford LD: Predictors of hearing preservation after stereotactic radiosurgery for acoustic neuroma. J Neurosurg 111: 863-873, 2009

5) Myrseth E, Møller P, Pedersen PH, Lund-Johansen M: Vestibular schwannoma: surgery or gamma knife radiosurgery? A prospective, nonrandomized study. Neurosurgery 64: 654-661; discussion 661-663, 2009

6) Tamura M, Carron R, Yomo S, Arkha Y, Muraciolle $\mathrm{X}$, Porcheron D, Thomassin JM, Roche PH, Régis J: Hearing preservation after gamma knife radiosurgery for vestibular schwannomas presenting with highlevel hearing. Neurosurgery 64: 289-296; discussion 296, 2009

7) Kim CH, Chung KW, Kong DS, Nam DH, Park K, Kim JH, Hong SH, Cho YS, Chung WH, Lee JI: Prognostic factors of hearing preservation after gamma knife radiosurgery for vestibular schwannoma. J Clin Neurosci 17: 214-218, 2010

8) Arthurs BJ, Fairbanks RK, Demakas JJ, Lamoreaux WT, Giddings NA, Mackay AR, Cooke BS, Elaimy 
AL, Lee CM: A review of treatment modalities for vestibular schwannoma. Neurosurg Rev 34: 265-277; discussion 277-279, 2011

9) Delbrouck C, Hassid S, Choufani G, De Witte O, Devriendt D, Massager N: Hearing outcome after gamma knife radiosurgery for vestibular schwannoma: a prospective Belgian clinical study. B-ENT 7(Suppl 17): 77-84, 2011

10) Massager N, Lonneville S, Delbrouck C, Benmebarek N, Desmedt F, Devriendt D: Dosimetric and clinical analysis of spatial distribution of the radiation dose in gamma knife radiosurgery for vestibular schwannoma. Int J Radiat Oncol Biol Phys 81: e511-e518, 2011

11) Sun S, Liu A: Long-term follow-up studies of Gamma Knife surgery with a low margin dose for vestibular schwannoma. J Neurosurg 117(Suppl): 57-62, 2012

12) Hasegawa T, Kida Y, Kato T, Iizuka H, Kuramitsu S, Yamamoto T: Long-term safety and efficacy of stereotactic radiosurgery for vestibular schwannomas: evaluation of 440 patients more than 10 years after treatment with Gamma Knife surgery. J Neurosurg 118: 557-565, 2013

13) Bir SC, Ambekar S, Bollam P, Nanda A: Long-term outcome of gamma knife radiosurgery for vestibular schwannoma. J Neurol Surg B Skull Base 75: 273-278, 2014

14) Boari N, Bailo M, Gagliardi F, Franzin A, Gemma M, del Vecchio A, Bolognesi A, Picozzi P, Mortini P: Gamma Knife radiosurgery for vestibular schwannoma: clinical results at long-term follow-up in a series of 379 patients. J Neurosurg 121(Suppl): 123-142, 2014

15) Lipski SM, Hayashi M, Chernov M, Levivier M, Okada Y: Modern Gamma Knife radiosurgery of vestibular schwannomas: treatment concept, volumetric tumor response, and functional results. Neurosurg Rev 38: 309-318; discussion 318, 2015

16) Régis J, Carron R, Park MC, Soumare O, Delsanti C, Thomassin JM, Roche PH: Wait-and-see strategy compared with proactive Gamma Knife surgery in patients with intracanalicular vestibular schwannomas. J Neurosurg 113 Suppl: 105-111, 2010

17) Hasegawa $T$, Kida $Y$, Kato T, Iizuka H, Yamamoto T: Factors associated with hearing preservation after Gamma Knife surgery for vestibular schwannomas in patients who retain serviceable hearing. J Neurosurg 115: 1078-1086, 2011

18) Han JH, Kim DG, Chung HT, Paek SH, Park CK, Kim CY, Kim YH, Kim JW, Kim YH, Song SW, Kim IK, Jung HW: Hearing preservation in patients with unilateral vestibular schwannoma who undergo stereotactic radiosurgery: reinterpretation of the auditory brainstem response. Cancer 118: 5441-5447, 2012

19) Kim JW, Kim DG, Paek SH, Chung HT, Kim YH, Han JH, Park CK, Jung HW: Efficacy of corticosteroids in hearing preservation after radiosurgery for vestibular schwannoma: a prospective study. Stereotact Funct Neurosurg 89: 25-33, 2011
20) Baschnagel AM, Chen PY, Bojrab D, Pieper D, Kartush J, Didyuk O, Naumann IC, Maitz A, Grills IS: Hearing preservation in patients with vestibular schwannoma treated with Gamma Knife surgery. $J$ Neurosurg 118: 571-578, 2013

21) Carlson ML, Jacob JT, Pollock BE, Neff BA, Tombers NM, Driscoll CL, Link MJ: Long-term hearing outcomes following stereotactic radiosurgery for vestibular schwannoma: patterns of hearing loss and variables influencing audiometric decline. $J$ Neurosurg 118: 579-587, 2013

22) Jacob JT, Carlson ML, Schiefer TK, Pollock BE, Driscoll CL, Link MJ: Significance of cochlear dose in the radiosurgical treatment of vestibular schwannoma: controversies and unanswered questions. Neurosurgery 74: 466-474; discussion 474, 2014

23) Yang I, Sughrue ME, Han SJ, Aranda D, Pitts LH, Cheung SW, Parsa AT: A comprehensive analysis of hearing preservation after radiosurgery for vestibular schwannoma. J Neurosurg 112: 851-859, 2010

24) Hayashi $M$, Chernov MF, Tamura N, Izawa $M$, Muragaki Y, Iseki H, Okada Y, Ivanov P, Régis J, Takakura K: Concept of robotic gamma knife microradiosurgery and results of its clinical application in benign skull base tumors. Acta Neurochir Suppl 116: 5-15, 2013

25) Yomo S, Tamura M, Carron R, Porcheron D, Régis $\mathrm{J}$ : A quantitative comparison of radiosurgical treatment parameters in vestibular schwannomas: the Leksell Gamma Knife Perfexion versus Model 4C. Acta Neurochir (Wien) 152: 47-55, 2010

26) Gardner G, Robertson JH: Hearing preservation in unilateral acoustic neuroma surgery. Ann Otol Rhinol Laryngol 97: 55-66, 1988

27) Koos WT, Day JD, Matula C, Levy DI: Neurotopographic considerations in the microsurgical treatment of small acoustic neurinomas. J Neurosurg 88: 506-512, 1998

28) Régis J, David P, Wikler D, Porcheron D, Levrier O: [Stereotactic mapping for radiosurgical treatment of vestibular schwannomas]. Neurochirurgie 50: 270-281, 2004 (French)

29) Wangerid T, Benmakhlouf H, Grane P, Bartek J Jr, Svensson M, Förander P: Implication of using MRI co-registered with CT in Leksell Gamma Knife(®) dose planning for patients with vestibular schwannoma. Clin Neurol Neurosurg 138: 10-15, 2015

30) Fukuoka S, Takanashi M, Hojyo A, Konishi M, Tanaka C, Nakamura H: Gamma knife radiosurgery for vestibular schwannomas. Prog Neurol Surg 22: 45-62, 2009

31) Massager N, Nissim O, Delbrouck C, Devriendt D, David P, Desmedt F, Wikler D, Hassid S, Brotchi J, Levivier M: Role of intracanalicular volumetric and dosimetric parameters on hearing preservation after vestibular schwannoma radiosurgery. Int J Radiat Oncol Biol Phys 64: 1331-1340, 2006

32) Massager N, Nissim O, Delbrouck C, Delpierre I, Devriendt D, Desmedt F, Wikler D, Brotchi J, Levivier M: Irradiation of cochlear structures during 
vestibular schwannoma radiosurgery and associated hearing outcome. J Neurosurg 107: 733-739, 2007

33) Linskey ME: Hearing preservation in vestibular schwannoma stereotactic radiosurgery: what really matters? J Neurosurg 109(Suppl): 129-136, 2008

34) Yomo S, Carron R, Thomassin JM, Roche PH, Régis J: Longitudinal analysis of hearing before and after radiosurgery for vestibular schwannoma. J Neurosurg 117: 877-885, 2012

35) Pollock BE, Link MJ, Foote RL, Stafford SL, Brown PD, Schomberg PJ: Radiosurgery as primary management for meningiomas extending into the internal auditory canal. Stereotact Funct Neurosurg 82: 98-103, 2004

36) Hasegawa T, Kato T, Kida Y, Hayashi M, Tsugawa T, Iwai Y, Sato M, Okamoto H, Kano T, Osano S, Nagano O, Nakazaki K: Gamma Knife surgery for patients with facial nerve schwannomas: a multiinstitutional retrospective study in Japan. J Neurosurg (in press)

37) Mousavi SH, Kano H, Faraji AH, Gande A, Flickinger JC, Niranjan A, Monaco E, Lunsford LD: Hearing preservation up to 3 years after gamma knife radiosurgery for Gardner-Robertson class I patients with vestibular schwannomas. Neurosurgery 76: 584-590; discussion 590-591, 2015
38) Kumon Y, Kohno S, Ohue S, Watanabe H, Inoue A, Iwata S, Ohnishi T: Usefulness of endoscope-assisted microsurgery for removal of vestibular schwannomas. J Neurol Surg B Skull Base 73: 42-47, 2012

39) Hori T, Maruyama T: Whether gamma knife radiosurgery is really necessary for treatment of patients with vestibular schwannomas. Acta Neurochir Suppl 116: 19-23, 2013

40) Régis J, Pellet W, Delsanti C, Dufour H, Roche PH, Thomassin JM, Zanaret M, Peragut JC: Functional outcome after gamma knife surgery or microsurgery for vestibular schwannomas. J Neurosurg 97: 1091-1100, 2002

41) Liu W, Ni M, Jia W, Zhou D, Zhang Q, Jiang Y, Jia G: How to address small- and medium-sized acoustic neuromas with hearing: a systematic review and decision analysis. World Neurosurg 84: 283-291. e1, 2015

Address reprint requests to: Motohiro Hayashi, MD, DMed Sci, Department of Neurosurgery, Neurological Institute, Tokyo Women's Medical University, 8-1 Kawada-cho, Shinjuku, Tokyo 162-8666, Japan. e-mail: GKRmoto@gmail.com 